\title{
OPTIC DISC EDEMA: AN INSIGHT TO THE INSIDE
}

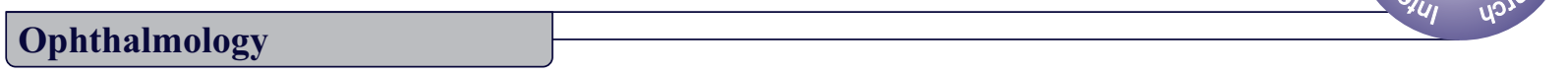

Dr Gayatri Associate Professor, Department of ophthalmology, MGM Medical College and Hospital, Bhonsale Navi Mumbai.

Dr Sayali Amberkar*

\section{Dr Ayushi Ojha}

Junior Resident, Department of ophthalmology, MGM Medical College and Hospital, Navi Mumbai. *Corresponding Author

Junior Resident, Department of ophthalmology, MGM Medical College and Hospital, Navi Mumbai.

\section{ABSTRACT}

The presentation of optic disc oedema could be secondary to an extensive set of underlying disorders, from infective to vascular to malignant.[1] Here we present 4 cases, each with a different underlying etiology, presenting to the emergency room (ER), whose careful history and complete evaluation led to finding a systemic cause which would have otherwise been missed. These cases need an extensive history, complete ocular examination and systemic evaluation to look for underlying etiologies. The series of cases presented here are meant to make the ophthalmology and the medicine residents more aware of the importance of a complete history and extensively examining a patient presenting to the ER with sudden loss of vision.

\section{KEYWORDS}

Optic Disc Edema, Systemic Lupus Erythematosus, Linezolid Induced Toxic Optic Neuropathy

\section{INTRODUCTION:}

A detailed history about the chief complaint, a systemic history to find the association if any and a complete ophthalmic examination is crucial to clinch the diagnosis. Optic disc edema depending on the time since it has developed may or may not affect the visual acuity, however an early diagnosis could lead to a better prognosis of any visual loss or associated systemic disorder. Presentation of a patient with optic disc edema could be due to a plethora of underlying disorders, including intrinsic ocular disease, intracranial lesions and systemic diseases. ${ }^{.2}$ Depending on the presentation being bilateral or unilateral, we can further pin point the differential Diagnosis. ${ }^{[3]}$

\section{CASE 1:}

\section{ACUTE BLURRING OF VISION IN ONE EYE}

\section{HISTORYAND EXAMINATION:}

45 year old male presented with the chief complaint of sudden painless and mildly progressive blurring of vision in the right eye (RE) since 5 days. The patient was not a known case of any systemic disorder and was not on any systemic medications.

On examination, visual acuity (VA) in RE was found to be $6 / 6$ for distance and N10 for near, and for the left eye (LE), 6/9 improving to $6 / 6$ for distance and N10 for near. Anterior segment was unremarkable and no pupillary abnormalities noted.

Dilated fundus examination with a direct ophthalmoscope(DO) showed optic disc edema in RE along with flame shaped and dot blot haemorrhages, with tortuosity of veins pointing towards the diagnosis of central retinal vein occlusion (CRVO)(Figure1), while the LE fundus was within normal limits (WNL) (Figure 2)

\section{COURSE AND OUTCOME:}

Given the age and the lack of any other clues an extensive systemic workup was done which showed positive Anti-Neutrophil Antibodies (ANA) and a rheumatology opinion was taken after which patient was diagnosed with Systemic Lupus Erythematosus(SLE) and was started on drugs accordingly. Disc edema resolved over a month.

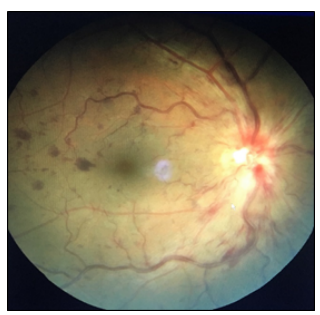

FIGURE 1

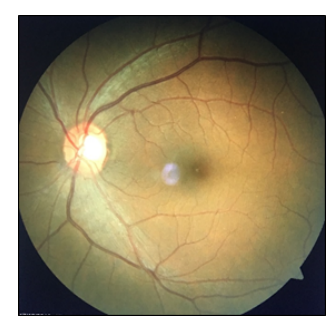

FIGURE 2

\section{CASE 2:}

\section{ACUTE PAINLESS VISION LOSS IN ONE EYE}

\section{HISTORY AND EXAMINATION:}

65 Year old male presented with chief complaint of sudden, painless progressive diminution of vision in LE since 20 days.

Patient had no known comorbidities

On examination, VA in the RE was $6 / 6$ for distance and $\mathrm{N} 6$ for near and $\mathrm{LE}$ counting finger at half metre for distance and $<\mathrm{N} 36$ for near. Pupils showed Grade 3 relative afferent pupillary defect (RAPD) in left eye and anterior segment showed both eyes (BE) pseudophakia and rest was unremarkable.

On dilated fundus examination with DO, RE was found within normal limits (Figure3) and LE showed optic disc edema (Figure 4).

\section{COURSE AND OUTCOME:}

After a systemic workup and investigations, the patient's blood sugars were raised and glycated haemoglobin $(\mathrm{HbAlC})$ was found to be 8.1 $\mathrm{mmol} / \mathrm{mol}$. Patient was diagnosed with optic neuritis secondary to uncontrolled diabetes mellitus (DM). The patient was commenced on a combination on a combination of insulin and oral anti diabetic drugs, after an urgent physician referral. Disc edema eventually resolved, after two weeks and VA in the LE improved to 6/18.

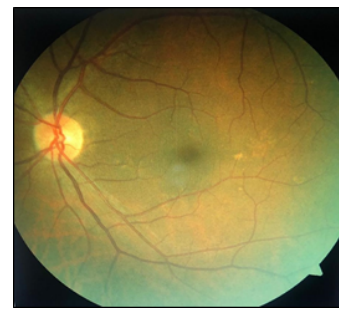

FIGURE 3

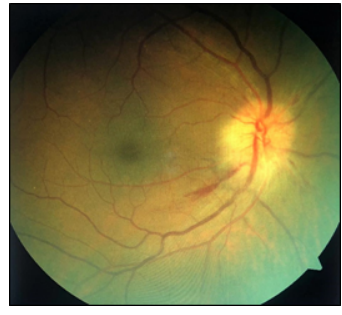

FIGURE 4

\section{CASE 3:}

\section{ACUTE BILATERAL DIMINUTION OF VISION}

\section{HISTORY AND EXAMINATION:}

A 17 year female presented with history of bilateral sudden painless progressive diminution of vision in BE since 1 week.

On examination: VA was found decreased in both eyes (distance and near), RE was 6/24 for distance and N10 for near and LE was 6/18 for distance and N10 for near. Colour vision was defective in both eye. 
Anterior segment was found normal with no pupillary abnormalities. Dilated fundus with DO revealed optic disc oedema in BE( Figures 5 and 6)

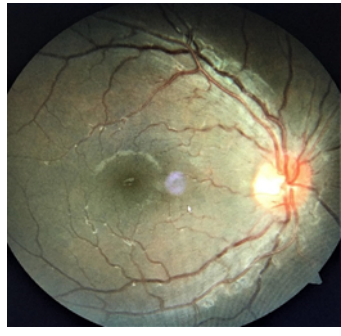

FIGURE 5

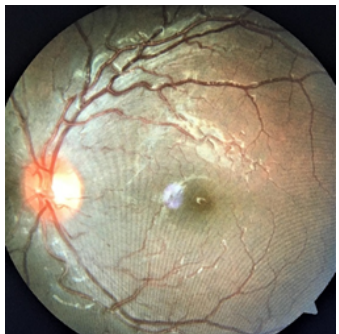

FIGURE 6
History of taking medications for multiple drug resistant-pulmonary tuberculosis (MDR-PTB) (second line since 7 months) was found.Patient was diagnosed with peripheral neuropathy following complaint of tingling and numbness in upper and lower limb, two months back.

\section{CLINICAL COURSE AND OUTCOME:}

Patient was diagnosed with bilateral toxic optic neuropathy as there was a positive history of patient being on second line treatment for MDR-PTB. A respiratory medicine referral was done to consider discontinuing linezolid The drug was stopped and the rest of the antitubercular drugs continued as previously. Disc oedema resolved gradually over 2 weeks and completely resolved over a month's time. The visual function showed a dramatic improvement with both eyes distance vision coming to $6 / 6$ and near vision coming N6, within a period of a month's time.

Colour vision did not return to normal in the early course of treatment, and was found normal on a follow up visit after 6 months.

\section{CASE 4}

\section{HISTORY AND EXAMINATION:}

A 48 year old patient presented to us with history of sudden, painless progressive diminution of vision in BE since 1 day. The patient had no known comorbidities.

On ophthalmic examination: VA was counting fingers at 2 metres not improving with pinhole and N36 in BE. Colour vision was defective and there was a grade 3 RAPD in left eye. Rest anterior segment was normal.

On dilated fundus examination with DO, Optic disc oedema with disc haemorrhage with attenuated vessels in RE, and Optic disc oedema with attenuated vessels in LE.

\section{On systemic examination:}

At presentation, blood pressure (BP) was found to be $200 / 100 \mathrm{~mm}$ of $\mathrm{Hg}$.

CLINICAL COURSE AND OUTCOME: Patient was found to be a hypertensive with current BP of 200/100 and was diagnosed with accelerated hypertension with hypertensive papillopathy. Patient was referred urgently to a physician and was started on anti-hypertensive medication. Disc Edema was gradually resolved and the visual acuity improved to $6 / 12$ in both eyes after the end of a month's period.

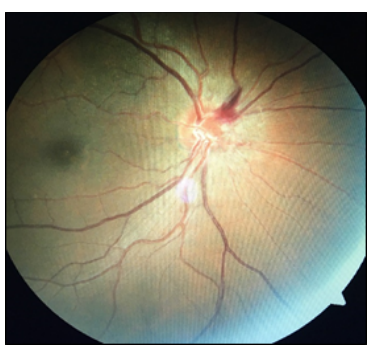

FIGURE 7

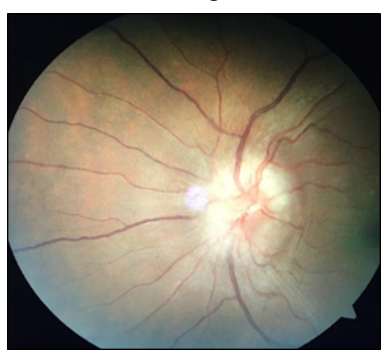

FIGURE 8

\section{DISCUSSION:}

Disc edema as observed in the above cases could be secondary to a diverse set of causes, and its diagnosis is key to an elaborate systemic examination to uncover the underlying disease. ${ }^{[2]}$
In the first case, given the patient's age and the sign of unilateral disc edema with haemorrhages with relatively preserved Visual acuity, and a positive ANA test, a diagnosis of Non ischaemic CRVO secondary to SLE, was made. A CRVO can be ischaemic and non ischaemic and can accordingly present with a certain degree of monocular visual loss, which is painless. ${ }^{[4]}$ As opposed to an ischaemic CRVO, which presents with profound visual loss, a non ischaemic CRVO, presents with a certain reduction in vision. ${ }^{[4]}$ Although in both of them, all four retinal quadrants are involved, The fundus picture is relatively less extreme in a non ischaemic CRVO ${ }^{[5]}$ Patients are usually over the age of 50 . In this particular case, the vascular abnormality was secondary to vascular changes due to underlying undiagnosed SLE. The vascular changes are due to the immune complexes deposited in the microvasculature of retina. ${ }^{[6]}$ Neuro-ophthalmic complications of SLE, although rare, could be sight threatening. A complete systemic workup ruled out all other causes except an auto immune disease, hence investigations were done accordingly.

In the second case, the patient had no known comorbidities and the presence of disc edema was the only presentation to warrant investigations. On finding a raised $\mathrm{HbA1c}$, a diagnosis of optic neuritis secondary to uncontrolled DM was made.. After this further tests were done to exclude microvascular complications and a possibility of end organ damage. So it warranted a series of tests to unravel the involvement of other organs, including kidneys. Laboratory investigations showed elevated serum creatinine levels. Diabetic papillopathy is a relatively rare condition associated with both type 1 and type 2 diabetes and can present with both unilateral and bilateral disc edema. ${ }^{[7}$

In the third case, the patient's young age, a prior history of being on second line anti tubercular treatment (ATT) with deficient colour vision, a diagnosis of Toxic optic neuropathy was made. The toxic optic neuropathy, secondary to AT drugs is a dose and duration dependent one, with the dyschromatopsia, which was observed in this patient, being the earliest and sometimes the only sign.

Classically toxic neuropathy presents with normal or minimally oedematous disc with minimal disc margin elevation and vascular changes and they may remain so till later stages.Early in the disease, optic nerves usually appear normal or, on occasion, slightly hyperemic. ${ }^{[8,9]}$ Discontinuation of the offending drug is the mainstay of treatment. Patients usually have an excellent prognosis with the visual acuity improving to $6 / 6$ in a couple of months post the withdrawal.

In the fourth case, the patient was not a known hypertensive and presented to the us with the clinical picture as above. A diagnosis of accelerated hypertension with hypertensive papillopathy was made, and the patient started on the necessary treatment by the physician.

As seen in all the above cases, patients presenting with sudden decreased vision or sudden loss of vision need a complete systemic workup in addition to a complete history and ocular examination. In many of these patients, visual loss maybe the only tell tale sign of a underlying systemic disease which maybe vision threatening or life threatening. Also patients who are on multiple systemic medications for TB/MDR-TB may develop vision threatening side effects which if diagnosed early could be reversible, therefore, the vision monitoring in this group is important

\section{CONCLUSION:}

The eyes are not only a window into one's soul but quite literally windows into one's body.

In our case series each case's initial presentation happened to be a visual loss and no other systemic symptoms. Complete ophthalmic examination, showed variable vision loss with optic disc edema. All the patients had an underlying systemic condition which was diagnosed after a thorough history, examination and systemic workup. Disc edema was diagnosed in all cases after a direct ophthalmoscopy done in the ER. Direct ophthalmoscopy is a vital skill for all the doctors working in the ER. Hence every patient presenting to the ER with visual loss and found to be having disc edema, must undergo an extensive history and complete systemic evaluation, which can sometimes help to diagnose even life threatening conditions.

\section{REFERENCES:}

1. https://www.aao.org/eyenet/article/case-studies-of-optic-disc-edema 
2. Kline LB. The swollen optic disc In: Kline LB, ed. Neuro-ophthalmology Review Manual. 6th Thorofare, NJ: SLACK Incorporated; 2007:139-152.

3. Hata M, Miyamoto K. Causes and Prognosis of Unilateral and Bilateral Optic Disc Swelling. Neuroophthalmology. 2017;41(4):187-191. Published 2017 Apr 10. doi:10.1080/01658107.2017.1299766

4. Karia N. Retinal vein occlusion: pathophysiology and treatment options. Clin Ophthalmol. 2010;4:809-816. Published 2010 Jul 30. doi:10.2147/opth.s7631

5. Kanski's Ophthalmology, 8th edition, Elsevier

6. Neal V. Palejwala, Harpreet S. Walia, Steven Yeh, "Ocular Manifestations of Systemic Lupus Erythematosus: A Review of the Literature", Autoimmune Diseases, vol. 2012, Article ID 290898, 9 pages, 2012. https://doi.org/10.1155/2012/290898

7. I.R. Wallace, D.A. Mulholland, J.R. Lindsay, Diabetic papillopathy: an uncommon cause of bilateral optic disc swelling, QJM: An International Journal of Medicine, Volume 105, Issue 6, June 2012, Pages 583-584, https://doi.org/10.1093/qjmed/hcr062

8. Margolin E, Shemesh A. Toxic and Nutritional Optic Neuropathy. [Updated 2020 Jul 5]. In: StatPearls [Internet]. Treasure Island (FL): StatPearls Publishing; 2020 Jan-. Available from: https://www.ncbi.nlm.nih.gov/books/NBK499979/

9. Karuppannasamy D, Raghuram A, Sundar D. Linezolid-induced optic neuropathy. Indian J Ophthalmol. 2014;62(4):497-500. doi:10.4103/0301-4738.118451

10. Modi P, Arsiwalla T. Hypertensive Retinopathy. [Updated 2020 Jul 10]. In: StatPearls [Internet]. Treasure Island (FL): StatPearls Publishing; 2020 Jan-. Available from: https://www.ncbi.nlm.nih.gov/books/NBK525980/ 